\title{
Evaluation of blood pressure responses to treadmill exercise test in normotensive children of hypertensive parents
}

\author{
Gökmen Özdemir ${ }^{1 \oplus}$, Pelin Köşger ${ }^{2 \oplus}$, Birsen Uçar ${ }^{2 \oplus}$ \\ ${ }^{1}$ Department of Pediatric Cardiology, Antalya Training and Research Hospital, Antalya; ${ }^{2}$ Department of Pediatric Cardiology, Eskişehir \\ Osmangazi University Faculty of Medicine, Eskişehir, Turkey.
}

\begin{abstract}
Background. Hypertension is a progressive disease with a prehypertensive phase. The most important feature of this period is the abnormal cardiovascular reactivity to various stressors. In our study, we focused on normotensive children of hypertensive parents, a special group that is at risk.

Methods. We evaluated the children according to age, studying whether they showed higher cardiovascular reactivity at different steps of an exercise test and during a recovery period than their counterparts with a negative history. A total of 110 normotensive children who were between the ages of 6 and 18 years were enrolled into the study. Sixty-two children whose parent(s) had a history of hypertension formed the study group while 48 without this history formed the control group. An exercise test was performed according to the Bruce protocol. Maximal systolic blood pressures and systolic blood pressures were taken while the participants were at rest; during phases 2 and 3; and also in the first, third, and sixth minutes of the recovery period.
\end{abstract}

Results. Measurements were significantly higher in the study group $(\mathrm{p}<0.05)$. In the group of children between the ages of 6 and 10 years, cardiovascular responses were similar. Children older than 10 years, however, had significantly higher blood pressure levels than those in the control group. The children who were at risk of hypertension showed more exaggerated cardiovascular responses during the exercise test and recovery period. This response was particularly evident for those children 10 years of age and older.

Conclusions. Our study indicates that treadmill exercise is a safe and effective investigational method which can be used to identify children who are at risk for development of hypertension before this condition becomes clinically evident.

Key words: cardiovascular physiological processes, exercise test, blood pressure, risk factors.

Primary or essential hypertension (HT) is a condition of elevated blood pressure (BP) with no identifiable underlying cause. ${ }^{1}$ Although genetic predisposition, environmental factors and role of the life style are considered in the etiopathogenesis of primary $\mathrm{HT}$, the pathophysiological process is highly complex and not completely understood. ${ }^{2}$ In many studies, it is found that genetic predisposition especially positive family history is a significant risk factor in the development of HT. ${ }^{3,4}$

\section{Gökmen Özdemir}

gokmenozdemir76@gmail.com

Received 7th January 2020, revised 18th March 2020, accepted 20th April 2020.
Although cardiac stress test is not easily available as a routine clinical tool used for diagnosing HT, it is a relatively cheap cardiovascular stimulation modality and frequently used for evaluating many clinical conditions. During the test, cardiovascular response is evaluated by monitoring electrocardiographic (ECG) traces and BP. A physiological response to the exercise is characterized by increased cardiac output, which is obtained by increasing heart rate and stroke volume, mediated by sympathetic system, in order to supply increased metabolic need of muscles. The BP response, secondary to the increased cardiac output, is compensated, albeit partially, by minimal decrease in peripheral vascular resistance. ${ }^{5}$ 
It is suggested that onset of the HT is associated with a pre-hypertensive course, which is primarily characterized with abnormal cardiovascular reaction to various stressors. ${ }^{6}$ It is also reported that patients with positive family history of HT usually have hyperactive sympathetic nervous system, and that hyperactive reactions lead to peripheral vasoconstriction, increased heart rate and ultimately, increase in peripheral vascular resistance. ${ }^{7}$ Moreover, it was found that there is a significant correlation between exaggerated $\mathrm{BP}$ reaction to the exercise test and increased peripheral vascular resistance caused by vascular endothelial dysfunction and arterial stiffness. ${ }^{8}$ Exaggerated response to cardiac stress test is also observed in hypertensive people and normotensive people with hypertensive parents. ${ }^{9,10}$

Evaluating BP response during exercise enables healthcare providers to identify children at risk for HT; this cannot be determined while they are at rest. ${ }^{11}$ However, the range of normal $\mathrm{BP}$ response that occurs during exercise in children and adolescents hasn't been clearly established. Most previous studies of BP levels during exercise have only involved investigations of the adult population, and their authors highlighted different stages of exercise testing and suggested various methodologies. ${ }^{11,12}$ For example, recent adult studies have been focused on the recovery period of the test, which has proven prognostic value. ${ }^{13,14}$ On the other hand, studies of this type conducted on children are scarce and most of them have only involved examination of maximal systolic and diastolic BP readings during exercise..$^{15,16}$ Therefore, pediatric cardiac stress test studies are not only limited in number but they also lack methodological quality.

Since it is known that pathophysiological processes of HT begin in childhood, we think there might be a relationship between a child's age, their stage of development, and these processes. Therefore, in our study, we focused on normotensive children of hypertensive parents (NCHP), a special at-risk group, to see if NCHP have higher cardiovascular reactivity in different steps of exercise testing and during a recovery period, as compared to their counterparts whose parents do not have HT.

\section{Material and Methods}

This was a cross-sectional study conducted with 110 normotensive children $\left(\mathrm{BP}<90^{\text {th }}\right.$ percentile according to age, gender and height), aged between 6-18 years, undergoing treadmill exercise testing according to Bruce protocol. Sixty-two children with parental history of HT formed NCHP group while age- and gendermatched 48 healthy children without parental history formed the control group (normotensive children of normotensive parents, NCNP). All subjects drafted from children who presented with silent murmur or non-specific chest pain. BP was measured with digital sphyngomanometer after the child rested for 10 minutes. Each measurement was repeated thrice, and the mean value was recorded. The family history of HT was defined as at least one parent who was diagnosed by a physician and requiring regular antihypertensive therapy. Normotensive parents were selected from those who had no history of HT and whose BPs were checked during the last 12 months by a physician. But the parents whose blood pressures were not checked at that time, their BPs were measured at our clinic. Children with chronic systemic disease, anemia or who were on medications that could interfere with the behavior of arterial BP and children with body mass index $>85^{\text {th }}$ percentile (with regards to age and gender) were not included. Families who refused cardiac stress test and children who would not possibly comply with the exercise test were also excluded. Children and their families were duly informed about the study and cardiac stress test and informed consent were taken. Participants were assigned to 1 of 3 groups according to age: 6 to 10 years (prepubertal); 11 to 14 years (pubertal); and 15 to 18 years (postpubertal).

Thestudy was approved by the Ethics Committee 
of the School of Medicine, Eskişehir Osmangazi University (Letter No. 28.04.2011/05). Informed consent was obtained from all individual participants included in the study.

\section{Treadmill test (cardiac stress testing)}

At treadmill test laboratory, ECG electrodes were placed according to Mason-Likar technique and a proper sized Orbit-K ${ }^{\mathrm{TM}} \mathrm{BP}$ cuff was fitted on the right arm of children and then they were allowed to rest for at least 10 minutes. ${ }^{17}$ Resting (basal) ECG, BP and heart rate were measured and recorded. The stress test was performed with General Electric Marquette Case Exercise Testing System (CASE Value v6.6 package) and GE T2100 treadmill (GE Healthcare Company, Wauwatosa, WI, USA). All participants did the treadmill exercise test using Bruce Protocol, a safe and standard stress protocol. The Bruce Protocol is a multiphase protocol, where inclination and velocity of the band are increased at each phase, and it is comprised of three-minute periods, where the patient is allowed to reach steady state, before workload is increased for the next phase. The test launched and ECG, BP and heart rate were recorded at second minutes of each threeminute stages during the test. The second part of the test was the recovery period or slowing down period and directly follows the exercise termination wherein the speed will decrease successively for a few minutes. Heart rate, ECG and BP were recorded at first, third and sixth minutes of this period. In our laboratory, BP is automatically measured by SunTech Tango + Stress BP monitor (SunTech Medical, Inc. Morrisville, NC, USA) with oscillation technique. The device is specifically developed for exercise testing and Korotkoff sounds, sensed by microphones equipped on orbit-K cuff, are analyzed by the developer company's patented Dimensional K-Sound Analysis "DKA".

Submaximal exercise (sub), implies the phase when a subject cannot yet reach the maximal exercise capacity in the exercise test and it is assigned as a pre-determined point or the duration the subject cannot exceed $85 \%$ of the maximal target heart rate. ${ }^{18}$ In our study, submaximal exercise response was identified as the end of second phase, since all children achieved this phase and had not yet reached $85 \%$ of the maximal heart rate. ${ }^{6,18,19}$

\section{Statistical analysis}

The statistical program IBM SPSS v20 for Windows was used to perform the statistical analysis. Normality of the distribution for all the variables was assessed with the Shapiro-Wilk test. Various variables which were normally distributed between the two study groups were compared using t-test, multiple comparisons of the variables were made by ANOVA test. Tukey's and Tamhane's post-hoc analysis was used to identify significant group differences that were indicated by ANOVA. For parameters without normal distribution, Mann-Whitney U test was used to compare two groups, while multiple comparisons were made with Kruskal-Wallis test. For analysis of cross tables, chi-square test was used. Pearson's correlation coefficients were computed to evaluate strength and direction of relationship of the measured variables showing normal distribution. A p-value of 0.05 or less was considered statistically significant. All data were expressed as mean \pm standard deviation (SD).

\section{Results}

Anthropometric data and clinical characteristics of NCHP and NCNP are given in Table I. Distribution of subjects by age groups is given in Table II. No statistically significant difference was found between groups in terms of age, gender, height, weight and BMI ( $p>0.05)$. Also there were no statistically significant differences between groups for exercise test duration and MET (metabolic equivalent, a MET is a ratio of working metabolic rate relative to resting metabolic rate. Average oxygen consumption in resting and sitting position is $3.5 \mathrm{ml} / \mathrm{kg} /$ min and this is expressed as " 1 MET") values. Maternal HT was noted in 37 children in the 
Table I. Comparison of baseline characteristics and exercise times between normotensive children of hypertensive parents and normotensive children of normotensive parents.

\begin{tabular}{lccc}
\hline & $\mathrm{NCHP}(\mathrm{n}=62)$ & $\mathrm{NCNP}(\mathrm{n}=48)$ & $\mathrm{p}$ \\
\hline Gender (female /male), $\mathrm{n}(\%) / \mathrm{n}(\%)$ & $28(45) / 34(55)$ & $22(46) / 26(54)$ & 0.94 \\
Age (years) & $12.11 \pm 3.35$ & $11.50 \pm 3.40$ & 0.94 \\
Weight $(\mathrm{kg})$ & $43.0 \pm 14.6$ & $38.9 \pm 14.6$ & 0.15 \\
Height $(\mathrm{cm})$ & $153.1 \pm 16.6$ & $148.2 \pm 20.3$ & 0.17 \\
Body mass index $\left(\mathrm{kg} / \mathrm{m}^{2}\right)$ & $17.67 \pm 2.80$ & $17.03 \pm 2.31$ & 0.21 \\
Endurance time $(\mathrm{min})$ & $12.48 \pm 1.83$ & $12.72 \pm 1.76$ & 0.48 \\
MET (metabolic equivalent) & $14.99 \pm 2.36$ & $15.03 \pm 2.12$ & 0.92 \\
\hline
\end{tabular}

Values are shown as mean \pm standard deviation or numbers (\%).

MET (metabolic equivalent): A MET is a ratio of working metabolic rate relative to resting metabolic rate. Average oxygen consumption in resting and sitting position is $3.5 \mathrm{ml} / \mathrm{kg} / \mathrm{min}$ and this is expressed as "1 MET", NCHP: normotensive children of hypertensive parents, NCNP: normotensive children of normotensive parents.

Table II. Distribution of children by age groups.

\begin{tabular}{lcc}
\hline Age groups & $\mathrm{NCHP}, \mathrm{n}(\%)$ & $\mathrm{NCNP}, \mathrm{n}(\%)$ \\
\hline $6-10$ ages & $22(35.5)$ & $18(37.5)$ \\
$11-14$ ages & $22(35.5)$ & $16(33.3)$ \\
$15-18$ ages & $18(29.0)$ & $14(29.2)$ \\
\hline Total & $62(100.0)$ & $48(100.0)$ \\
\hline
\end{tabular}

NCHP: normotensive children of hypertensive parents, NCNP: normotensive children of normotensive parents.

NCHP group (59.6\%), while 25 fathers (40.4\%) were hypertensive in the same group. None of the children had a history of HT in both parents.

Resting systolic blood pressure (SBP) records were significantly higher in the NCHP group than in the NCNP group ( $\mathrm{p}=0.001)$. However, no inter-group difference was found for diastolic blood pressure (DBP) or heart rate (HR). During the test, phase-2 SBP, phase-3 SBP, and phase- 4 DBP readings of NCHP were significantly higher than those of the NCNP group $(p=$ $0.001, p=0.009$, and $p=0.04$, respectively). SBP readings measured at the first, third, and sixth minutes of recovery were significantly higher in the NCHP group in comparison to those of the control group $(\mathrm{p}=0.02, \mathrm{p}=0.001$, and $\mathrm{p}=$ 0.001 , respectively). No statistically significant difference was found between the groups in terms of heart rate and DBP during the recovery period $(p>0.05)$. Changes in SBP, DBP, and heart rate readings of both groups are shown in Figure 1.
Maximal and submaximal (phase-2) BP and HR values and their changes according to the resting values are summarized in Table III . Maximal SBP responses were significantly higher in the NCHP group than in the NCNP group ( $p=$ 0.007). No difference was found between the two groups in terms of changes in HR and BP readings relative to resting values $(p>0.05)$.

When exercise data were compared by gender, test durations (13.23 vs. $11.82 \mathrm{~min}$ ) and MET values (15.91 vs. 13.92) were found to be significantly higher in male subjects. In the NCHP group, resting HR and phase-2 HR (sub$\mathrm{HR}$ ) measurements were higher in females than males ( $p=0.04$ and $p=0.01$ ) (Fig. 2a). Moreover, maximal SBP $(p=0.03)$ (Fig. $2 b), \triangle \max -\mathrm{SBP}(\mathrm{p}=$ $0.01), \Delta$ max-DBP $(\mathrm{p}=0.02)$, and $\Delta$ sub-SBP $(\mathrm{p}=$ $0.04)$ readings were significantly higher in male subjects than in those of the females (data not shown). But in the NCNP group, no difference was found between the genders in all variables, excluding higher MET values and longer test durations of the males. 
Table III. Maximal and submaximal (phase 2) blood pressure and heart rate values and their changes according to resting values.

\begin{tabular}{lccc}
\hline Parameters & NCHP & NCNP & $p$ \\
\hline sub-SBP & $142.1 \pm 16.1$ & $132.4 \pm 11.3$ & 0.001 \\
sub-DBP & $65.5 \pm 10.7$ & $63.0 \pm 8.5$ & 0.188 \\
$\Delta$ sub-SBP & $29.3 \pm 14.7$ & $26.4 \pm 11.4$ & 0.25 \\
$\Delta$ sub-DBP $\alpha$ & $2.5[-8.0 ; 9.0]$ & $1.0[-4.0 \pm(+6.5)]$ & 0.79 \\
max-SBP & $162.3 \pm 21.1$ & $152.7 \pm 13.6$ & 0.007 \\
max-DBP & $69.2 \pm 10.4$ & $65.8 \pm 7.8$ & 0.061 \\
$\Delta$ max-SBP & $49.7 \pm 18.9$ & $46.7 \pm 14.5$ & 0.054 \\
$\Delta$ max-DBP & $4.66 \pm 11.03$ & $4.7 \pm 9.2$ & 0.36 \\
sub-HR & $140.6 \pm 17.7$ & $137.4 \pm 14.4$ & 0.31 \\
$\Delta$ sub-HR & $39.1 \pm 15.1$ & $38.2 \pm 14.2$ & 0.75 \\
max-HR & $194.3 \pm 10.8$ & $192.3 \pm 11.8$ & 0.33 \\
$\Delta$ max-HR & $92.9 \pm 18.0$ & $93.1 \pm 20.0$ & 0.95 \\
\hline
\end{tabular}

Values are shown as mean \pm standard deviation or median $\left[25^{\text {th }}\right.$ percentile; $75^{\text {th }}$ percentile].

DBP: diastolic blood pressure, HR: heart rate, max: maximal, NCHP: normotensive children of hypertensive parents, NCNP: normotensive children of normotensive parents, SBP: systolic blood pressure, sub: submaximal (phase 2), $\Delta$ sub: submaximal-resting value, $\Delta$ max: maximal-resting value.

When data were compared by age, no statistically significant difference was found in any group among the NCNP and NCHP groups in terms of exercise duration and MET values ( $\mathrm{p}$ $>0.05$ ). No statistically significant difference was found between subgroups of those who were 6 to 10 years of age in terms of exercise test data points ( $\mathrm{p}>0.05)$. For the subgroups of children ages 11 to 14 , resting-DBP $(\mathrm{p}=0.04)$, phase- 1 SBP $(p=0.04)$, phase-2 SBP $(p=0.017)$, phase- 3 DBP $(p=0.016)$, phase-4 DBP $(p=0.03)$, maxDBP $(p=0.006)$, R1-DBP $(p=0.04)$ and R3-SBP $(\mathrm{p}=0.04)$ readings were significantly higher in the NCHP group than in the NCNP group. For the subgroup with children ages 15 to 18 , resting SBP $(p=0.006)$, phase-1 SBP $(p=0.038)$, phase-2 SBP $(p=0.006)$, phase-3 SBP $(p=0.002)$, phase-4 SBP $(p=0.009), \max \operatorname{SBP}(p=0.002), R 1$ SBP ( $p=0.024)$, R3 SBP ( $p=0.004)$, and R6 SBP $(p=0.001)$ measurements were significantly higher in the NCHP group in comparison to those of the NCNP group (Fig. 3).

Changes observed in SBP and DBP readings by age subgroups during the exercise test and recovery period are shown in Figure 3 (A-B-C).
For paternal or maternal HT we found all the test results to be statistically similar ( $p>0.05$ ). When age and anthropometric data of children in the study group were correlated with data obtained during the exercise test and recovery period, resting $\mathrm{SBP}$, maximal $\mathrm{SBP}, \mathrm{HR}$ at the first minute, and SBP at the sixth minute of the recovery period showed strong correlations with age, height, weight, and BMI .

We observed no problem or complication, such as severe arrhythmia, dyspnea and excessive hypertensive response (>250/125 $\mathrm{mmHg})$, which would, otherwise, require terminating the test.

\section{Discussion}

\section{Pre-stress test data}

In this study, the resting SBP levels of NCHP were significantly higher than those of the control group. We believe that this finding can be related to increased sympathetic stimuli secondary to mental stress, although no physical stress was observed in that group. It is suggested that the onset of HT is associated with a pre-hypertensive course, primarily 


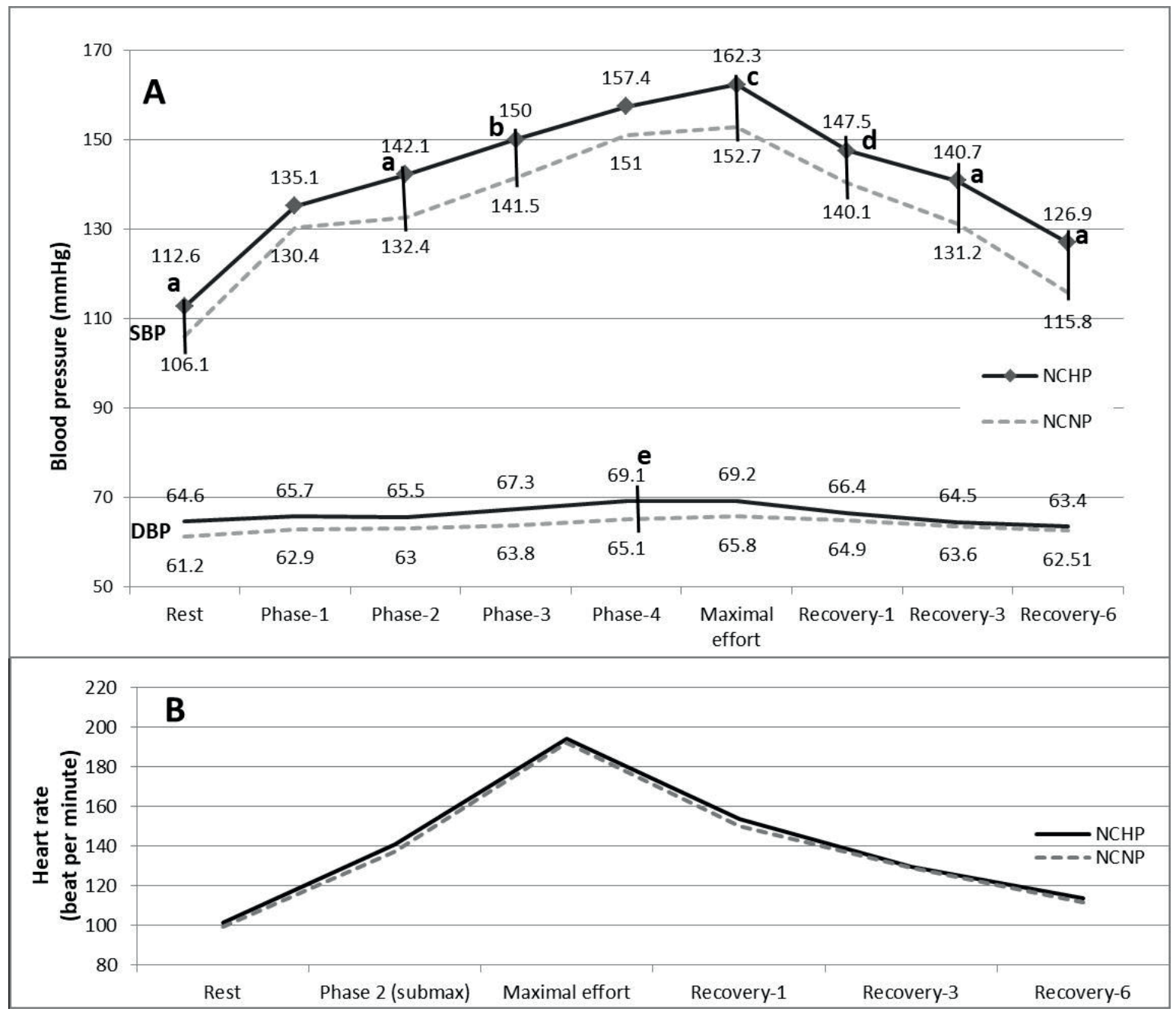

Fig. 1. Graphical presentation of blood pressure (A) and heart rate (B) values obtained during exercise test and recovery period in the study and control groups.

Letters ( $a, b, c, d, e)$ indicate specific phase or period at which the difference between groups is statistically significant ( $p$ $<0.05$ ).

DBP: diastolic blood pressure, NCHP: normotensive children of hypertensive parents, NCNP: normotensive children of normotensive parents; recovery $1-3-6,1^{\text {st }}, 3^{\text {rd }}$ and $6^{\text {th }}$ minutes of recovery period; SBP: systolic blood pressure. ${ }^{a} p=0.001,{ }^{b} p=$ $0.009,{ }^{c} \mathrm{p}=0.007,{ }^{\mathrm{d}} \mathrm{p}=0.02,{ }^{\mathrm{e}} \mathrm{p}=0.04$

characterized by abnormal cardiovascular reactions to various stressors. ${ }^{6}$ Varying sensitivity levels of adrenergic receptors and sympathetic hyperactivity are suggested for exaggerated cardiovascular response to the stress. ${ }^{20}$ Sympathetic hyperactivity is secondary to excessive sympathetic stimuli and/or decreased sympathetic inhibition. ${ }^{21}$ This hyperactivity starts in childhood and can be demonstrated in $30 \%$ of recently diagnosed hypertensive subjects. ${ }^{20}$ Sowmya et al. ${ }^{22}$ conducted a study on NCHP and found that their resting SBP was higher than that of control subjects. Similar to our study, the authors didn't observe a difference in DBP or HR responses. Carroll et al. ${ }^{23}$ found a correlation between prestress resting SBP and SBP measured at year-5 of the follow-up; this was in the Whitehall 2 study, in which the authors also determined that mental stress reactivity was insufficient for identifying future HT. 


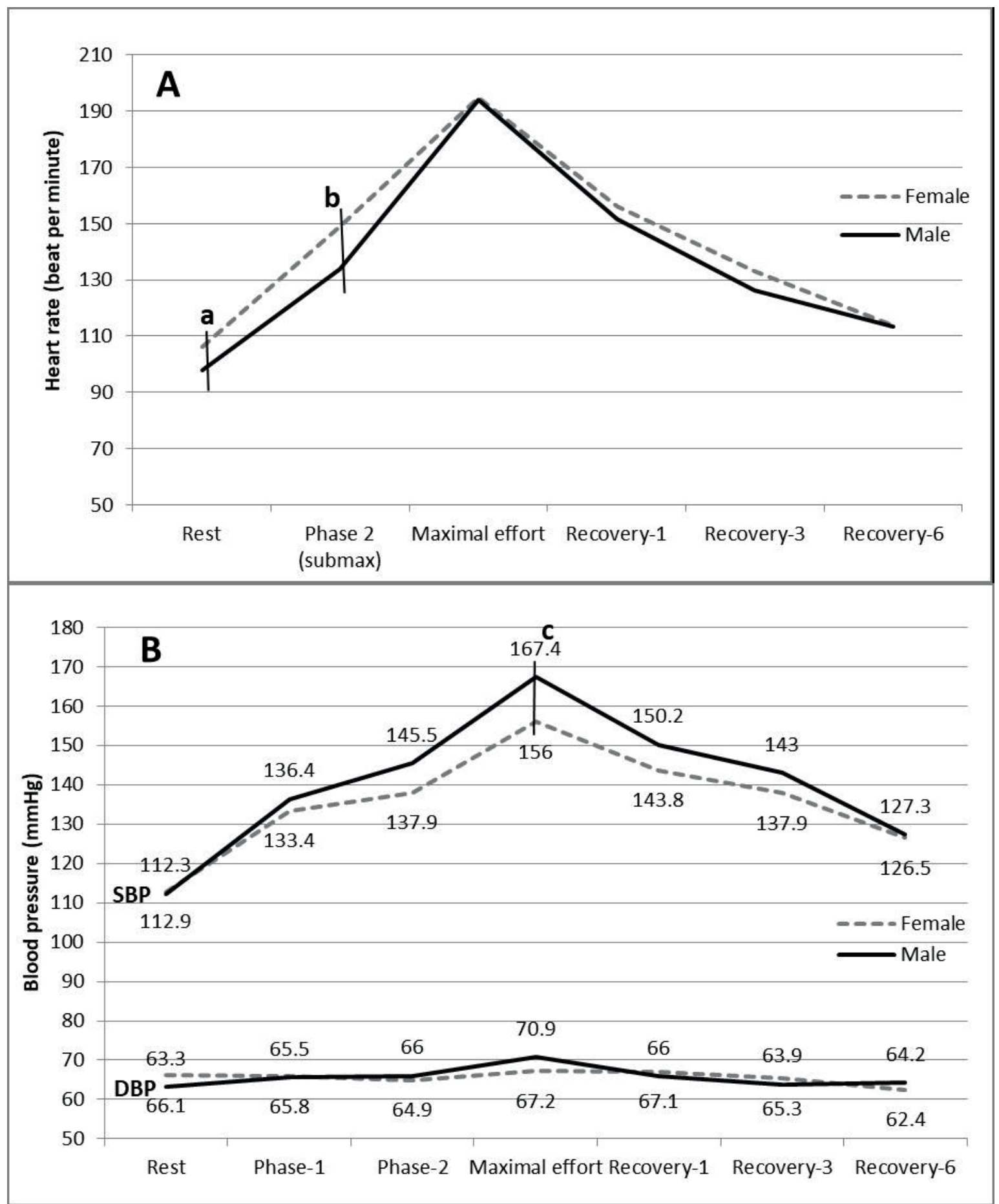

Fig. 2. Graphical presentation of heart rate (A) and blood pressure (B) values obtained during exercise test and recovery period in the study group by gender.

Letters $(a, b, c)$ indicate specific phase or period at which the difference between groups is statistically significant $(p<0.05)$.

DBP: diastolic blood pressure; recovery $1-3-6,1^{\text {st }}, 3^{\text {rd }}$ and $6^{\text {th }}$ minutes of recovery period; SBP: systolic blood pressure. ${ }^{\mathrm{a}} \mathrm{p}=0.04$, ${ }^{b} \mathrm{p}=0.01,{ }^{c} \mathrm{p}=0.03$ 


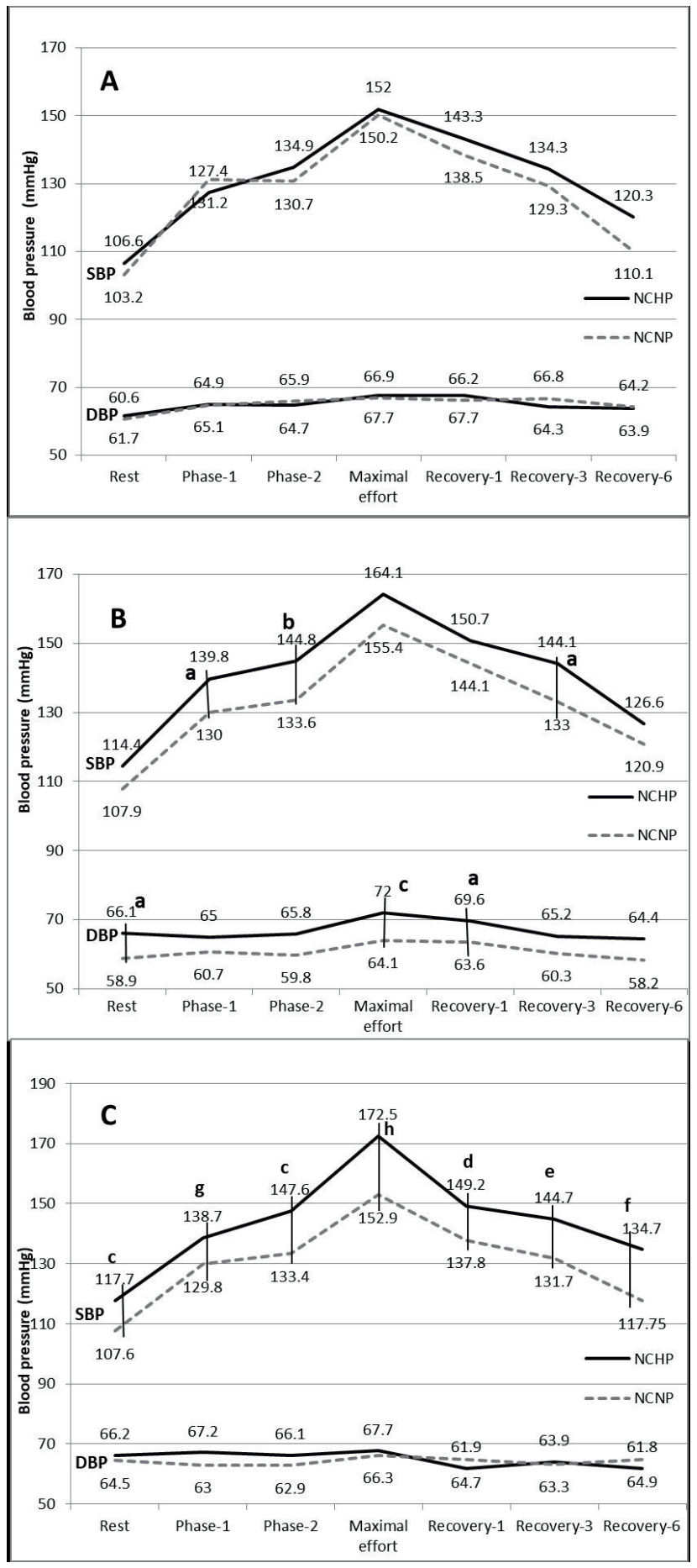

Fig. 3. Graphical presentation of systolic and diastolic blood pressure readings obtained during exercise test and recovery period in six-to-ten years (A), eleven-to-fourteen years (B), fifteen-to-eighteen years (C) of age subgroups.

Letters ( $a, b, c, d, e, f, g, h)$ indicate specific phase or period at which the difference between groups is statistically significant ( $p<0.05)$.

DBP: diastolic blood pressure, NCHP: normotensive children of hypertensive parents, NCNP: normotensive children of normotensive parents; recovery $1-3-6,1^{\text {st }}, 3^{\text {rd }}$ and $6^{\text {th }}$ minutes of recovery period, SBP: systolic blood pressure. ${ }^{\text {a }} \mathrm{p}=0.04$, ${ }^{\mathrm{b}} \mathrm{p}=$ $0.017,{ }^{c} \mathrm{p}=0.006,{ }^{\mathrm{c}} \mathrm{p}=0.006,{ }^{\mathrm{g}} \mathrm{p}=0.038 .{ }^{\mathrm{h}} \mathrm{p}=0.002,{ }^{\mathrm{d}} \mathrm{p}=0.024,{ }^{\mathrm{e}} \mathrm{p}=0.004,{ }^{\mathrm{f}} \mathrm{p}=0.001$ 
Adult studies like that of Miyai et al. ${ }^{6}$ conducted on normotensive men have shown that finding an individual relative risk for developing HT in normotensive adults with slightly elevated resting $\mathrm{BP}$ is greatly increased if they exhibit an exaggerated BP response to exercise. These results indicate that the measurement of exercise BP can provide some additional important information concerning the risk for developing HT that cannot be estimated by resting $\mathrm{BP}$ alone. The authors also stated that measurements of resting $\mathrm{BP}$ often show spurious elevated values because of anxiety, which decreases the reproducibility of results and their usefulness for predicting future HT.

\section{Cardiac stress test data}

During the exercise test, phase-2 SBP, phase-3 SBP, phase-4 DBP, and maximal SBP were significantly higher in the NCHP group than in the control group. Wilson et al. ${ }^{24}$ determined that although there was no inter-group difference in measurements such as resting BP, vascular resistance, and cardiac output, an exaggerated response $(\geq 230 / 100 \mathrm{mmHg}$ ) to the cardiac stress test was found in $35 \%$ of the members of the group with a positive family history and a high normal resting BP. However, there was no standard definition of hypertensive response for a normotensive and asymptomatic subject. Various models were suggested by the authors of different studies. In a study conducted on an adult population, it was determined that for subjects with a BP response of $\geq 225 / 90 \mathrm{mmHg}$ in a treadmill exercise test, the risk of HT was 2.3fold greater at the end of a 32-month period. ${ }^{25}$ As suggested by this study and others that are similar to it, the most commonly suggested model is maximal SBP (max-SBP) during maximal exercise. ${ }^{26}$ Some authors consider that $\mathrm{BP}$ responses recorded during submaximal exercise are of clinical significance. ${ }^{19}$ The principle reason is that maximal SBP depends on high-level exercise and the physical condition of the subject. ${ }^{8}$ Singh et al. ${ }^{19}$ examined adult participants of the Framingham study and demonstrated that exaggerated DBP measured at phase 2 (submaximal phase) of the stress test and exaggerated SBP measured at the third minute of the recovery period were valuable in estimating the development of HT. Schultz et al. ${ }^{27}$ conducted a meta-analysis by reviewing adult studies and observed that independent of age, outpatient BP values, and other risks, hypertensive response to submaximal exercise was related to cardiovascular events and mortality. They also stated that the prevalence of masked HT reaches up to $58 \%$ in such subjects. We found that both submaximal (phase 2) SBP responses and maximal SBP responses were significantly higher in the NCHP; this finding is supportive of data from those studies.

In some studies, researchers have used extra variables, along with gender and percentile values, when BP responses of subjects were evaluated. For example, Miyai et $a .^{6}$ used the heart rate reserve measurement to evaluate the influence of the same metabolic load on BP. Similarly, the change in BP per minute $(\mathrm{mmHg} / \mathrm{min})$, the change in SBP relative to resting $\mathrm{BP}(\triangle \mathrm{SBP})$, and the max $\mathrm{DBP}$ have been used in various studies. ${ }^{26,28-31}$ Several authors have suggested that BP behavior and $\mathrm{BP}$ variability observed during the stress test, rather than maximal $\mathrm{BP}$, are more important for determining the risk of HT. Although we examined changes in SBP and DBP relative to resting $\mathrm{BP}$ between two groups, both in phase 2 (submaximal exercise) and maximal exercise, no significant difference was found in terms of those variables $(p>0.05)$. However, $\triangle \max S B P$ values of the NCHP were higher than those of subjects in the control group, although this finding was not statistically significant $(p=$ 0.054).

\section{Recovery period data}

During the recovery period, SBP responses at the first, third, and sixth minutes were found to be significantly higher in the NCHP group. Nakashima et al. ${ }^{32}$ examined responses of the study population (mean age, 19 years) to the submaximal exercise test and demonstrated that SBP and DBP values recorded for male 
subjects immediately after exercise were strong predictors of future HT. Yosefy et al. ${ }^{13}$ used the Bruce exercise protocol for study groups and found a relationship between $\mathrm{BP} \geq 160 / 90 \mathrm{mmHg}$ at the fifth minute of the recovery period and a poor cardiac profile. Similarly, Tsumura et al. ${ }^{33}$ conducted a study in which they followed 6557 normotensive adults and found a correlation between each $10 \mathrm{mmHg}$ increase in SBP and DBP measured at the fourth minute of the recovery period and a 1.55-fold relative risk of HT, while Laukkanen et al. ${ }^{34}$ linked SBP $\geq 195 \mathrm{mmHg}$ at the second minute of the recovery period in the study group to an increased risk of myocardial infarction. In this study, we evaluated three stages of the recovery period, including early (first minute) and late (sixth minute) phases of the test, rather than just a single phase. We observed that the SBP response was high in the NCHP group in all three phases of the recovery. But the DBP and HR responses were similar in both groups.

American College of Cardiology and American Heart Association guidelines acknowledges max-SBP $\geq 214 \mathrm{mmHg}$ and SBP and DBP measured at third minutes of the recovery period - based on the estimated relative risk found in the Framingham Study - as indicators of the possible future HT. ${ }^{19,35}$

\section{Evaluation of data by age groups, gender and hypertension of the parents}

Considering our study data, it was observed that children up to 10 years of age had similar cardiovascular reactivity in both groups. But children with a family history of HT showed higher BP responses after 10 years of age. At rest, in the group of 11- to 14-year-olds, a difference was observed in DBP. In older children the difference in SBP responses became remarkable. SBP responses in the first two phases of the exercise test (including the submaximal phase) were significantly higher in children older than 10 years of age. DBP responses were even higher in the group of 11- to 14-year-olds during phases 3,4 , and 5 and during maximal exercise, while elevated SBP was predominant in those 15 years of age and older during those phases and during maximal exercise. Similarly, in the group of 11- to 14-year-olds, a significant difference in DBP responses was observed in the early phase of recovery. SBP responses were higher in the third minute of recovery in the NCHP group. In the group of 15- to 18-yearolds, elevated SBP responses at the first, third, and sixth minutes of recovery were the primary findings in the NCHP group.

We observed various results upon the review of studies which analyzed changes in the BP profiles of children of hypertensive parents over the course of time. In their first study, Li et al. ${ }^{36}$ monitored third graders for 5 years and found that the NCHP group showed higher levels of resting $\mathrm{BP}$ and increased cardiovascular reactivity than the other children. However, they showed cardiovascular reactivity only in SBP change. van Hooft et al. ${ }^{37}$ studied BP and related characteristics in children whose parents both had relatively high BP. The results were compared to those of children whose parents both had a relatively low BP; they were also compared to those of children who had one parent with high BP and one parent with low BP. At the age of 8 years there were no clear differences in $\mathrm{BP}$ but at the age of 20 years there was a difference of $7 \mathrm{mmHg}$ for both systolic and diastolic pressure between the high- and low-risk offspring.

In the Bogulasa study, when compared to 5- to 9-year-olds, a stronger relationship was found between BP monitoring of 10- to 14-year-olds and HT in adult years. ${ }^{38}$ Also, the effects of parental HT on longitudinal changes in BP have been examined by Mitsumata et al. ${ }^{39}$ They stated that the parallel shift of the age-BP relationship by parental HT suggests that a set point of $\mathrm{BP}$ is elevated by parental HT before the third decade of life. In our study, we demonstrated increased cardiovascular responses in children older than 10 years of age with family history but who were still normotensive. Moreover, increased cardiovascular reactivity became explicit first in DBP responses after 10 years of age and then in SBP responses after the age of 14 years. An 
increased DBP response to an exercise test can be explained by increased peripheral vascular resistance and impaired capacity for exerciseinduced vasodilatation. Our results support that increased peripheral vascular resistance may begin at younger ages when children are still normotensive and have normal SBP responses to exercise.

Cardiac output increase during exercise is a function of heart rate $x$ stroke volume. Due to physiological differences between genders, it has been suggested that men have a greater reliance on preload and an enhanced use of the Frank-Starling mechanism whereas females rely on heart rate to increase cardiac output. ${ }^{40,41}$ During exercise, an increase in sympathetic activity and a decrease of vagal discharge leads to an increase of heart rate, stroke volume, and myocardial contractility to satisfy energy demands of working skeletal muscles. Therefore, the regulation and homeostatic relationship between BP and cardiac output is achieved through different mechanisms in males and females. ${ }^{40,41}$ We found that resting and phase-2 HRs were higher in girls than boys, but maximal SBPs and their changes $(\Delta \max$, $\Delta$ sub) according to resting levels were observed as statistically higher in boys.

Fomin et al..$^{40}$ analyzed adolescents' responses to a maximal exercise test and observed that girls had higher levels of resting HR and lower levels of resting SBP while boys displayed higher levels of maximal SBP responses. In the same study, maximal HRs and resting DBP responses of both genders were found to be similar. Dimkpa et al. ${ }^{41}$ measured maximal SBP and DBP with SBP responses at the first minute of recovery as being higher in boys; on the other hand, they measured maximal HR and SBP responses at the third minutes of recovery and found the results to be similar in both genders. In the same study, maximal SBP changes $(\Delta \max )$ were found to be higher in boys compared to resting BP measurements. However, in contradistinction to the findings of the studies mentioned above, in the study of Akdur et al. ${ }^{42}$ conducted on healthy Turkish children, this difference wasn't seen between the two genders with regard to maximal HR and SBP. But a linear correlation was observed between max SBP, age, and exercise duration.

By power analysis, for variables other than submax HR, it was noted that studies need to be conducted in larger groups to make a comparison of females and males involving the $\mathrm{BP}$ response to the exercise test.

Among the 62 children who constituted the NCHP group, mothers of 37 children and fathers of 25 children had HT. When the exercise test data were compared according to parent HT status, there was no statistical significance between the children whose mother or father had HT. There are different studies in which the leaders have investigated the effect of maternal and paternal BP on childhood BP. For example, it was shown that the hazard ratio of HT development was 1.5 for men with maternal HT only; 1.8 for men with paternal HT only; and 2.4 for men with HT in both parents. ${ }^{43}$ On the other hand, DeStefano et al. ${ }^{44}$ argued that a mother with HT is a stronger risk factor. But, similar to our study, Mitsumata et al. ${ }^{39}$ observed that there was no statistical difference in children's HT development with regard to the mother or father being hypertensive.

The measurement of maximal DBP levels is usually difficult because of technical difficulties and because of noise during the test. Therefore, this is excluded in the majority of studies and SBP responses are the only ones examined. This issue becomes more obvious in clinics where BP is measured manually during the test. However, automated instruments provide more precise DBP and SBP values and more studies on this are underway. Cameron et al. ${ }^{45}$ compared data of automated BP assessments using the Tango exercise BP monitor (SunTech Medical Instruments, NC, USA) - the same instrument as the one we used in our study - with data gathered via an invasive method. They found that the results were similar and within a reliable clinical range. Consequently, we think the systolic and diastolic BP values measured in 
our study are close to the most accurate values.

It was found that in both the recovery period and during the test, the children with parental HT had more excessive cardiovascular responses to the physical stress than the children without parental HT. These responses were more prominent in children older than 10 years of age. Furthermore, at that age, increased cardiovascular reactivity became explicit first in DBP response and then in SBP response. Because of different periods of physical development, defining a threshold level for hypertensive responses in children is very challenging and a larger pediatric population is needed for study. Therefore, we did not aim to give a threshold level for hypertensive responses.

Our data were obtained cross-sectionally from a relatively small group of children. However, we think that our results can be useful in designing future longitudinal studies in a larger pediatric population. By power analysis, it was considered that the sample sizes of the age groups of NCHP and NCNP were sufficient for comparisons of the $\mathrm{BP}$ response to the exercise test among members of the groups. But for variables other than submax (phase 2) HR, it was seen that there is a need for studies to be done in larger groups to make a comparison between females and males of $\mathrm{BP}$ response to an exercise test.

Overweight and obese children were not included in the study. We also didn't evaluate sports or leisure time physical activities and lifestyles of the children enrolled in the study.

At the time of this investigation, the BP of some normotensive parents were not measured and the declaration of these families were taken into account.

The exercise test may not be a diagnostic tool for HT, but it can be used to identify patients with abnormal BP responses that may be a precursor to HT. This has the potential to be used for intervention and possibly to prevent or delay the development of HT by the early identification of individuals who are at risk, so that they may introduce modifications to their diet and lifestyle. We believe our study will help expand knowledge on this subject as studies in children are relatively few and many of them have flaws in their methodology.

\section{Acknowledgments}

The authors would like to thank technician Umran Ozyurek for her help with data acquisition. Also, we thank Cengiz Bal, Ph.D., for his assistance with statistical analysis.

\section{REFERENCES}

1. Falkner B. Hypertension in Children and Adolescents. In: Moller J, Hoffman J (eds). Pediatric Cardiovascular Medicine (2nd ed). West Sussex: Wiley-Blackwell; 2012; 938-953.

2. Hamet P, Pausova Z, Adarichev V, Adaricheva K, Tremblay J. Hypertension: genes and environment. J Hypertens 1998; 16: 397-418.

3. van den Elzen AP, de Ridder MA, Grobbee DE, Hofman A, Witteman JC, Uiterwaal CS. Families and the natural history of blood pressure. A 27-year follow-up study. Am J Hypertens 2004; 17: 936-940.

4. Hong Y, de Faire U, Heller DA, McClearn GE, Pedersen N. Genetic and environmental influences on blood pressure in elderly twins. Hypertension 1994; 24: 663-670.

5. Pianosi P, Driscoll D. Exercise esting. In: Allen HD, Driscoll DJ, Shaddy RE (eds): Moss and Adam's Heart Disease in Infants, Children and Adolescents (8th ed) Philadelphia: Lippincott Williams \& Wilkins (LWW); 2013; 118-133.

6. Miyai N, Arita M, Miyashita K, Morioka I, Shiraish T, Nishio I. Blood pressure response to heart rate during exercise test and risk of future hypertension. Hypertension 2002; 39: 761-766.

7. Julius S, Schork MA. Predictors of hypertension. Ann NY Acad Sci 1978; 304: 38-58.

8. Thanassoulis G, Lyass A, Benjamin EJ et al. Relations of exercise blood pressure response to cardiovascular risk factors and vascular function in the Framingham Heart Study. Circulation 2012; 125: 2836-2843.

9. Herkenhoff FL, Vasquez EC, Mill JG, Lima EG. Ambulatory blood pressure and Doppler echocardiographic indexes of borderline hypertensive men presenting an exaggerated blood pressure response during dynamic exercise. Braz J Med Biol Res 2001; 34: 1285-1293. 
10. Ziada AM, Al Kharusi W, Hassan MO. Exaggerated blood pressure reactivity in the offspring of firstcousin hypertensive parents. J Sci Res Med Sci 2001; 3: 81-85.

11. Molineux D, Steptoe A. Exaggerated blood pressure responses to submaximal exercise in normotensive adolescents with a family history of hypertension. J Hypertens 1988; 6: 361-365.

12. Sieira MC, Ricart AO, Estrany RS. Blood pressure response to exercise testing. Apunts Med Esport 2010; 45: 191-200.

13. Yosefy C, Jafari J, Klainman E, Brodkin B, Handschumacher MD, Vaturi M. The prognostic value of post-exercise blood pressure reduction in patients with hypertensive response during exercise stress test. Int J Cardiol 2006; 111: 352-357.

14. Huang CL, Su TC, Chen WJ, et al. Usefulness of paradoxical systolic blood pressure increase after exercise as a predictor of cardiovascular mortality. Am J Cardiol 2008; 102: 518-523.

15. Becker Mde M, Barbosa e Silva O, Moreira IE, Victor EG. Arterial blood pressure in adolescents during exercise stress testing. Arq Bras Cardiol 2007; 88: 329-333.

16. Yilmaz M, Pirim Ü, Çetin M, Kiremitçi S, Coşkun S. Ailede hipertansiyon öyküsü olan çocukların egzersiz testi yanıtları. Turkiye Klinikleri J Pediatr 2013; 22: 1-7.

17. Mason RE, Likar I. A new system of multiple-lead exercise electrocardiography. Am Heart J 1966; 71: 196-205.

18. Paridon SM, Alpert BS, Boas SR, et al; American Heart Association Council on Cardiovascular Disease in the Young, Committee on Atherosclerosis, Hypertension, and Obesity in Youth. Clinical stress testing in the pediatric age group: a statement from the American Heart Association Council on Cardiovascular Disease in the Young, Committee on Atherosclerosis, Hypertension, and Obesity in Youth. Circulation 2006; 113: 1905-1920.

19. Singh JP, Larson MG, Manolio TA, et al. Blood pressure response during treadmill testing as a risk factor for new-onset hypertension. The Framingham heart study. Circulation 1999; 99: 1831-1836.

20. Mills PJ, DimsdaleJE, Nelesen RA, Jasiewicz J, Zeigler MG, Kennedy B. Patterns of adrenergic receptors and adrenergic agonists underlying cardiovascular responses to a psychological challenge. Psychosom Med 1994; 56: 70-76.

21. Julius S. The evidence for a pathophysiologic significance of the sympathetic overactivity in hypertension. Clin Exp Hypertens 1996; 18: 305-321.
22. Sowmya R, Maruthy KN, Gupta R. Cardiovascular autonomic responses to whole body isotonic exercise in normotensive healthy young adult males with parental history of hypertension. Indian J Physiol Pharmacol 2010; 54: 37-44.

23. Carroll D, Smith GD, Sheffield D, Shipley MJ, Marmot MG. Pressor reactions to psychological stress and prediction of future blood pressure: data from Whitehall II Study. BMJ 1995; 310: 771-776.

24. Wilson MF, Sung BH, Pincomb GA, Lovallo WR. Exaggerated pressure response to exercise in men at risk for systemic hypertension. Am J Cardiol 1990; 66: 731-736.

25. Wilson NV, Meyer BM. Early prediction of hypertension using exercise blood pressure. Prev Med 1981; 10: 62-68.

26. Sharabi Y, Ben-Cnaan R, Hanin A, Martonovitch G, Grossman E. The significance of hypertensive response to exercise as a predictor of hypertension and cardiovascular disease. J Hum Hypertens 2001; 15: 353-356.

27. Schultz MG, Otahal P, Cleland VJ, Blizzard L, Marwick TH, Sharman JE. Exercise-induced hypertension, cardiovascular events, and mortality in patients undergoing exercise stress testing: a systematic review and meta-analysis. Am J Hypertens 2013; 26: 357-366.

28. Kurl S, Laukkanen JA, Rauramaa R, Lakka TA, Sivenius J, Salonen JT. Systolic blood pressure response to exercise stress test and risk of stroke. Stroke 2001; 32: 2036-2041.

29. Matthews CE, Pate RR, Jackson KL, et al. Exaggerated blood pressure response to dynamic exercise and risk of future hypertension. J Clin Epidemiol 1998; 51: 29-35.

30. Ha JW, Juracan EM, Mahoney DW, et al. Hypertensive response to exercise: a potential cause for new wall motion abnormality in the absence of coronary artery disease. J Am Coll Cardiol 2002; 39: 323-327.

31. Zanettini JO, Fuchs FD, Zanettini MT, Zanettini JP. Is hypertensive response in treadmill testing better identified with correction for working capacity? A study with clinical, echocardiographic and ambulatory blood pressure correlates. Blood Pressure 2004; 13: 225-229.

32. Nakashima M, Miura K, Kido T, et al. Exercise blood pressure in young adults as a predictor of future blood pressure: a 12-year follow-up of medical school graduates. J Hum Hypertens 2004; 18: 815821. 
33. Tsumura K, Hayashi T, Hamada C, Endo G, Fujii S, Okada K. Blood pressure response after two-step exercise as a powerful predictor of hypertension: the Osaka Health Survey. J Hypertens 2002; 20: 15071512.

34. Laukkanen JA, Kurl S, Salonen R, Lakka TA, Rauramaa R, Salonen JT. Systolic blood pressure during recovery from exercise and the risk of acute myocardial infarction in middle-aged men. Hypertension 2004; 44: 820-825.

35. Gibbons RJ, Balady GJ, Bricker JT, et al; American College of Cardiology/American Heart Association Task Force on Practice Guidelines. Committee to Update the 1997 Exercise Testing Guidelines ACC/ AHA 2002 guideline update for exercise testing: summary article. A report of the American College of Cardiology/American Heart Association Task Force on Practice Guidelines (Committee to Update the 1997 Exercise Testing Guidelines). J Am Coll Cardiol 2002; 40: 1531-1540.

36. Li R, Alpert BS, Walker SS, Somes GW. Longitudinal relationship of parental hypertension with body mass index, blood pressure, and cardiovascular reactivity in children. J Pediatr 2007; 150: 498-502.

37. van Hooft IM, Hofman A, Grobbee DE, Valkenburg HA. Change in blood pressure in offspring of parents with high or low blood pressure: the Dutch Hypertension and Offspring Study. J Hypertens Suppl 1988; 6: S594-S596.

38. Bao W, Threefoot SA, Srinivasan SR, Berenson GS. Essential hypertension predicted by tracking of elevated blood pressure from childhood to adulthood: the Bogalusa Heart Study. Am J Hypertens 1995; 8: 657-665.
39. Mitsumata K, Saitoh S, Ohnishi H, Akasaka H, Miura T. Effects of parental hypertension on longitudinal trends in blood pressure and plasma metabolic profile: mixed-effects model analysis. Hypertension 2012; 60: 1124-1130.

40. Fomin $\AA$, Ahlstrand M, Schill HG, et al. Sex differences in response to maximal exercise stress test in trained adolescents. BMC Pediatr 2012; 12: 127.

41. Dimkpa U, Ugwu AC, Oshi DC. Assessment of sex differences in systolic blood pressure responses to exercise in healthy, non-athletic young adults. J Exerc Physiol Online 2008; 11: 18-25.

42. Akdur $H$, Sözen AB, Yiğit Z, Oztunç F, Kudat $H$, Güven $\mathrm{O}$. The evaluation of cardiovascular response to exercise in healthy Turkish children. Turk J Pediatr 2009; 51: 472-477.

43. Wang NY, Young JH, Meoni LA, Ford DE, Erlinger TP, Klag MJ. Blood pressure change and risk of hypertension associated with parental hypertension: the Johns Hopkins Precursors Study. Arch Intern Med 2008; 168: 643-648.

44. DeStefano AL, Gavras H, Heard-Costa N, et al. Maternal component in the familial aggregation of hypertension. Clin Genet 2001; 60: 13-21.

45. Cameron JD, Stevenson I, Reed E, McGrath BP, Dart AM, Kingwell BA. Accuracy of automated auscultatory blood pressure measurement during supine exercise and treadmill stress electrocardiogram-testing. Blood Press Monit 2004; 9: 269-275. 